\title{
Impaired awareness of motor intention in functional neurological disorder: implications for voluntary and functional movement
}

\author{
K. Baek ${ }^{1}$ t, N. Doñamayor ${ }^{1}$ t, L. S. Morris ${ }^{2}$, D. Strelchuk ${ }^{1}$, S. Mitchell ${ }^{1}$, Y. Mikheenko ${ }^{1}$, S. Y. Yeoh ${ }^{3}$, \\ W. Phillips ${ }^{4}$, M. Zandi ${ }^{4,5,6}$, A. Jenaway ${ }^{7}$, C. Walsh ${ }^{1,4}$ and V. Voon ${ }^{1,2,7,8 *}$ \\ ${ }^{1}$ Department of Psychiatry, University of Cambridge, Cambridge, UK \\ ${ }^{2}$ Behavioural and Clinical Neurosciences Institute, Cambridge, UK \\ ${ }^{3}$ School of Clinical Medicine, University of Cambridge, Cambridge, UK \\ ${ }^{4}$ Department of Clinical Neurosciences, Addenbrooke's Hospital, Cambridge, Cambridge, UK \\ ${ }^{5}$ Department of Molecular Neuroscience, UCL Institute of Neurology, London, UK \\ ${ }^{6}$ National Hospital for Neurology and Neurosurgery, UCLH NIHR Biomedical Research Centre, London, UK \\ ${ }^{7}$ Cambridgeshire and Peterborough NHS Foundation Trust, Cambridge, UK \\ ${ }^{8}$ NIHR Biomedical Research Council, Cambridge, Cambridge, UK
}

Background. Functional neurological disorders (FNDs), also known as conversion disorder, are unexplained neurological symptoms unrelated to a neurological cause. The disorder is common, yet poorly understood. The symptoms are experienced as involuntary but have similarities to voluntary processes. Here we studied intention awareness in FND.

Method. A total of 26 FND patients and 25 healthy volunteers participated in this functional magnetic resonance study using Libet's clock.

Results. FND is characterized by delayed awareness of the intention to move relative to the movement itself. The reporting of intention was more precise, suggesting that these findings are reliable and unrelated to non-specific attentional deficits. That these findings were more prominent with aberrant positive functional movement symptoms rather than negative symptoms may be relevant to impairments in timing for an inhibitory veto process. Attention towards intention relative to movement was associated with lower right inferior parietal cortex activity in FND, a region early in the processing of intention. During rest, aberrant functional connectivity was observed with the right inferior parietal cortex and other motor intention regions.

Conclusions. The results converge with observations of low inferior parietal activity comparing involuntary with voluntary movement in FND, emphasizing core deficiencies in intention. Heightened precision of this impaired intention is consistent with Bayesian theories of impaired top-down priors that might influence the sense of involuntariness. A primary impairment in voluntary motor intention at an early processing stage might explain clinical observations of slowed effortful voluntary movement, heightened self-directed attention and underlie functional movements. These findings further suggest novel therapeutic targets.

Received 17 June 2016; Revised 3 January 2017; Accepted 4 January 2017; First published online 10 February 2017

Key words: Functional magnetic resonance imaging, functional neurological disorder, inferior parietal cortex, intention, voluntary action.

\section{Introduction}

Functional neurological disorder (FND), or conversion disorder, refers to neurological symptoms in the absence of a neurological condition (American Psychiatric Association, 2013). The disorder is common (Carson et al. 2000) and equally physically debilitating as

* Address for correspondence: V. Voon, M.D., Ph.D., Department of Psychiatry, University of Cambridge, Addenbrooke's Hospital, Level E4, Box 189, Cambridge CB2 0QQ, UK.

(Email: vv247@cam.ac.uk)

+ These authors contributed equally to the paper.
Parkinson's disease, with greater impact on mental health and quality of life (Anderson et al. 2007; Voon et al. 2016). Still, it remains poorly understood. Symptoms are experienced as involuntary but have similar physiological features as voluntary movements (Hallett, 2010). An early crucial study hypothesized that a disturbance of volition might underlie FND (Spence et al. 2000). Here we focus on the awareness of motor intention as one aspect of volition, using Libet's clock paradigm (Libet et al. 1983).

Libet et al. (1983) reported that awareness of the urge or intention to move ( $\mathrm{W}$ judgement) preceded awareness of the movement itself (M judgement) by around

This is an Open Access article, distributed under the terms of the Creative Commons Attribution licence (http://creative commons.org/licenses/by/4.0/), which permits unrestricted re-use, distribution, and reproduction in any medium, provided the original work is properly cited. 
$200 \mathrm{~ms}$. Intention awareness was itself preceded by the Bereitschaftspotential, i.e. readiness potential, suggesting an unconscious initiation of the volitional process and constraining the potential for action control (Libet et al. 1983; Libet, 1999). This has been widely replicated (Lau et al. 2004; Brass \& Haggard, 2007; Fried et al. 2011). However, Libet emphasized the potential role for action control and the relevance of the interval between $\mathrm{W}$ and $\mathrm{M}$ judgements in the veto hypothesis: despite unconscious action initiation, action control would be plausible in that interval, allowing for a veto or inhibitory process (Libet, 1999; Haggard \& Libet, 2001). Delays in $W$ relative to $M$ judgement have been reported in Parkinson's disease (Tabu et al. 2015) and Tourette's syndrome (Moretto et al. 2011), suggesting impairments in action control.

The supplementary motor complex (SMC) and inferior parietal cortex (IPC) have been identified as key regions underlying motor intention. Epilepsy patients reported the urge to move when supplementary and pre-supplementary motor areas (SMA, pre-SMA) were electrically stimulated (Fried et al. 1991), and single-neuron recordings showed increased firing as the W judgement approached (Fried et al. 2011). Attention to intention with Libet's clock also activates the pre-SMA, intraparietal sulcus and dorsolateral prefrontal cortex (dlPFC) (Lau et al. 2004). The IPC has been suggested to play a role upstream of the SMC in the development of motor intention with evidence from electrical stimulation, stroke lesion and motor imagery studies (Sirigu et al. 2004; Desmurget et al. 2009; Desmurget \& Sirigu, 2012).

Converging studies implicate impairments in explicit intentional processes in FND. An early study of functional paralysis demonstrated impaired dlPFC activity to attempted movement, which was linked to a disturbance of volition (Spence et al. 2000). Functional compared with voluntary tremor has been associated with right temporoparietal junction (TPJ)/ IPC hypoactivity, a region implicated in sensorimotor integration (Voon et al. 2010). The authors suggested a possible impairment of intentional or prediction processes, as sensory regions were intact. A follow-up study demonstrated SMA hypoactivity in FND subjects during motor preparation, as well as decreased dlPFC-SMA connectivity during internally generated v. externally cued action selection (Voon et al. 2011). Using Libet's clock, a small study reported that FND patients with functional tremor $(n=11)$ had significantly delayed $\mathrm{W}$ relative to $\mathrm{M}$ judgements compared with healthy volunteers (HV) (Edwards et al. 2011), such that the timing of both judgements was indistinguishable, implying abnormalities in the conscious experience of action underlying functional movements.
In this experiment, we aimed to explore awareness of voluntary motor intention in a larger sample of FND patients with mixed symptoms and HV. We acquired functional magnetic resonance images (fMRI) while participants performed the task devised by Libet et al. (1983). We hypothesized that FND patients would have delayed motor intention awareness, and lower IPC and SMC activity compared with HV.

\section{Method \\ Participants}

A total of $26 \mathrm{FND}$ patients and $25 \mathrm{HV}$ took part in the study, and one FND patient and one HV were later excluded due to interruption of the experiment during the scan. FND subjects were referred by neurologists and psychiatrists from Addenbrooke's Hospital, Cambridge, and recruited through the FND Hope website (http://fndhope.org/). The diagnosis was based on Diagnostic and Statistical Manual of Mental Disorders, Fifth edition (DSM-5) diagnostic criteria (American Psychiatric Association, 2013) and either made or confirmed by a neurologist from the FND clinic (W.P., M.Z.). Participants were screened by psychiatrists for psychiatric co-morbidities and to document symptom severity (S.M., V.V.). FND subjects with any other major neurological or psychiatric diagnosis, including current major depression greater than moderate severity [Beck Depression Inventory-II $($ BDI-II) $>17$ ], psychotic or bipolar disorder and substance use disorder were excluded from the study. Current mild depression and elevated depression scores with no current major depression diagnosis were allowed. Recruitment was limited by subjects with head movements, who had ballistic movements, were unable to remain still in the scanner or had claustrophobia. HV were recruited via community advertisements. Participants gave written informed consent and were reimbursed for their time. All experimental procedures were approved by the University of Cambridge Research Ethics Committee.

Participants completed the BDI-II (Beck et al. 1961) and Spielberger State-Trait Anxiety Inventory (STAI; Spielberger et al. 1983). Pain, motor (paralysis or weakness, non-epileptic seizures, tremor, chorea, tics, gait abnormalities, dystonia, myoclonus) and sensory (somatosensory, vision, hearing) symptoms were recorded based on clinical interview by systematic enquiry, and rating of duration and severity $[1=$ mild (limited impact on daily functioning); 2 =moderate (noticeable impact on daily functioning with restriction of some activities); 3 = severe (marked impact on daily functioning with restriction of activity in multiple 
domains); 4 =very severe (impairment in all or virtually all domains of activity)].

\section{Stimuli and procedure}

In the Libet's clock task (Libet et al. 1983; Lau et al. 2004) participants watched a red ball revolving around an unnumbered clock face at $2500 \mathrm{~ms}$ per cycle (Fig. 1a). Participants were instructed to press a button with their left index finger after a random time interval after waiting for one cycle. They were asked to act as spontaneously as possible and in particular to avoid preselecting a position of the ball to trigger the button press. If there was no button press within three cycles the trial was recorded as missed.

The task consisted of two types of trials. In intention trials, participants were asked to attend to when they felt the urge to press the button (W judgement). In movement trials, they were required to attend to when they actually pressed the button (M judgement). After the button press, the ball continued moving for 1000-2500 ms (0.4-1 revolution). Then, it appeared on a random angle on the clock face, and participants were required to return it to the position it was in when they first felt the urge to press the button, or actually pressed it, depending on the type of trial. The final position of the red ball was recorded if still for $2 \mathrm{~s}$ or after the $7 \mathrm{~s}$ period for responding elapsed. The inter-trial interval lasted 1-6 s. Average latencies of the $\mathrm{W}$ and $\mathrm{M}$ judgements were calculated as the differences between the final position of the ball and its position at the time of the recorded button press.

Prior to fMRI data acquisition, participants took part in a brief practice session of 10 movement followed by 10 intention trials. The fMRI task consisted of four blocks of 20 trials in the order of movement-intentionmovement-intention. The fMRI experiment lasted around 18-21 $\mathrm{min}$.

\section{MRI data acquisition}

MRI data were acquired with a $3 \mathrm{~T}$ Tim Trio scanner (Siemens, Germany) with a 32-channel head coil at the Wolfson Brain Imaging Centre, University of Cambridge. Foam pads were used to restrict head movements. Echo planar imaging (EPI) images were acquired in an interleaved fashion [repetition time $(\mathrm{TR})=2.32 \mathrm{~s}$, echo time $(\mathrm{TE})=30 \mathrm{~ms}, 3 \times 3 \times 3 \mathrm{~mm}$ voxel size, $0.75 \mathrm{~mm}$ gap, flip angle $(\mathrm{FA})=78^{\circ}, 64 \times 64$ matrix size, 39 slices]. Images were angled $30^{\circ}$ to the anterior commissure-posterior commissure line in order to avoid susceptibility signal loss in the orbitofrontal regions. The T1-weighted structural images were acquired using a magnetization-prepared rapid gradient-echo (MPRAGE) sequence $[\mathrm{TR}=2.30 \mathrm{~s}$, TE $=$
$2.98 \mathrm{~ms}$, field of view $(\mathrm{FOV})=176 \times 240 \mathrm{~mm}^{2}, 1 \times 1 \times 1$ $\mathrm{mm}^{3}$ voxel size, inversion time $=1100 \mathrm{~ms}$.

Resting-state fMRI (rsfMRI) data were acquired after the fMRI task for $10 \mathrm{~min}$ with open eyes. A multiecho EPI sequence was used with online reconstruction $\left[\mathrm{TR}=2.47 \mathrm{~s}, \mathrm{FA}=78^{\circ}\right.$, matrix size $64 \times 64,3.75 \mathrm{~mm}$ in-plane resolution, $\mathrm{FOV}=240 \mathrm{~mm}, 32$ oblique slices, alternating slice acquisition, slice thickness $3.75 \mathrm{~mm}$ with $10 \%$ gap, integrated parallel imaging technique (iPAT) factor 3, bandwidth $=1698 \mathrm{~Hz} /$ pixel, $\mathrm{TE}=12$, $28,44$ and $60 \mathrm{~ms}]$.

\section{fMRI data analysis}

fMRI data were analysed using SPM8 (http://www.fil. ion.ucl.ac.uk/spm/software/spm8/; Wellcome Trust Centre for Neuroimaging, UK). After slice-timing correction, a mean image for all functional scans was generated for each subject, to which individual volumes were spatially realigned by rigid body transformation. Unwarping was performed during realignment to correct for dynamic motion-distortion interaction artefacts. Structural images were co-registered with the mean EPI image, segmented into grey and white matter, and normalized to the Montreal Neurological Institute (MNI) template. Normalization parameters were then applied to the EPI images for an anatomically informed normalization. Images were then subsampled to $2 \times 2 \times 2 \mathrm{~mm}^{3}$ and spatially smoothed with a $10 \mathrm{~mm}$ full width at half maximum (FWHM) Gaussian filter. Scan-by-scan head motions exceeding $0.5 \mathrm{~mm}$ in the functional volumes were repaired using Art Repair (Mazaika et al. 2007).

The subject-level statistical analyses were performed using the general linear model (GLM). The main events of interest were the $1 \mathrm{~s}$ periods prior to the recorded button press in intention and movement trials. Missed trials and the $7 \mathrm{~s}$ period for responding were modelled as events of no interest. Vectors containing the event onsets and durations were convolved with the canonical haemodynamic response function, and its temporal and dispersion derivatives. The statistical parameter estimates were computed separately for each voxel for all 12 columns in the design matrix. Movement parameters were also included as regressors of no interest in the GLM in addition to deweighting on the repaired volumes.

Intention $v$. movement trials were contrasted at the individual level and subsequently inputted into an independent-samples $t$ test to compare FND patients and HV. Whole-brain voxel-wise group comparisons were performed with a cluster extent threshold of 15 voxels at $p<0.001$ (uncorrected), correcting for multiple comparisons at $p<0.05$ assuming an individualvoxel type I error of $p=0.01$ (Slotnick et al. 2003). We 

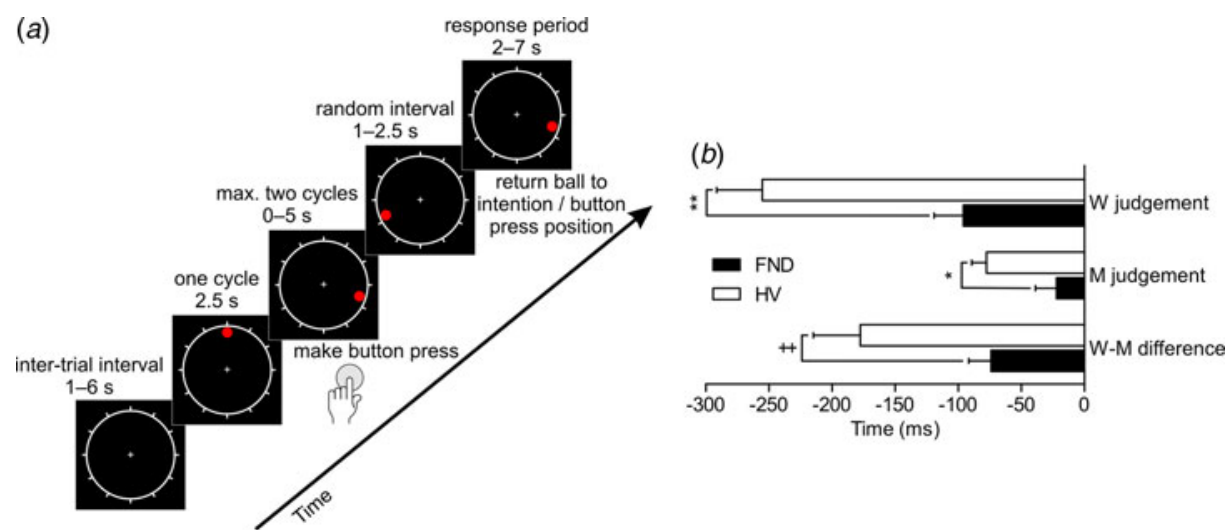

Fig. 1. Libet's clock task. (a) Schematic representation of the task. The red ball revolved around the unnumbered clock face for a maximum of three cycles; participants had to make a button press after waiting one cycle. The ball continued moving for a random interval, after which participants returned the ball to its position when they had felt the urge (W judgement) or actually pressed the button (M judgement). (b) Estimated times of intention (W judgement), movement (M judgement) and difference between intention and movement (W-M) relative to the recorded button press for functional neurological disorder (FND) patients and healthy controls (HV). Values are means, with standard errors represented by the horizontal bars. ${ }^{++} p=$ $0.017,{ }^{*} p=0.009,{ }^{* *} p=0.001$. For a colour figure, see the online version of the paper.

also confirmed fMRI activations in a priori regions of interest (ROIs) implicated in Lau et al. (2004), the pre-SMA, the inferior parietal lobule (IPL) and dlPFC, using small-volume correction as well.

The intention $v$. movement blood oxygen leveldependent (BOLD) contrast was also correlated with the behavioural parameter $\mathrm{W}-\mathrm{M}$ across all individuals.

\section{rsfMRI data analysis}

rsfMRI data were analysed with multi-echo independent component analysis (ME-ICAv2.5 beta6; http://afni.nimh.nih.gov). First, FastICA was used to decompose multi-echo fMRI data. The BOLD signal is characterized by TE-dependence, which was measured using the pseudo- $F$ statistic $\kappa$; whereas TE-independence was measured by the pseudo- $F$ statistic $\rho$. Components were then categorized as BOLD or non-BOLD based on their $\kappa$ - and $\rho$-value weightings, respectively (Kundu et al. 2012), and non-BOLD components were removed by projection. Denoised EPI images were co-registered to MPRAGE, normalized to the MNI template, spatially smoothed with a $6 \mathrm{~mm}$ FWHM Gaussian filter, and temporally bandpass filtered between 0.008 and $0.09 \mathrm{~Hz}$. Anatomical scans were segmented into grey matter, white matter and cerebrospinal fluid (CSF), and significant principal components in white matter and CSF regions were identified and removed following the CompCor strategy (Behzadi et al. 2007).

ROI-driven functional connectivity was computed with the CONN-fMRI Functional Connectivity toolbox (Whitfield-Gabrieli \& Nieto-Castañón, 2012) for SPM8. Based on the task-based results, the rsfMRI analysis was seeded within the right IPC, as defined in the Automated Anatomical Labelling atlas.

ROI-to-voxel whole-brain connectivity maps were computed and entered into full factorial GLMs to compare whole-brain connectivity patterns between FND patients and HV. Whole-brain voxel-wise group comparisons were performed with a cluster extent threshold of 15 voxels at $p<0.001$ (uncorrected), correcting for multiple comparisons at $p<0.05$ assuming an individual-voxel type I error of $p=0.01$ (Slotnick et al. 2003).

\section{Ethical standards}

All procedures contributing to this work comply with the ethical standards of the relevant national and institutional committees on human experimentation and with the Helsinki Declaration of 1975, as revised in 2008.

\section{Results \\ Participants}

In all, 25 FND patients and 24 age- and gendermatched HV completed the study. Participant characteristics are described in Table 1, with FND subjects displaying significantly higher depression and anxiety scores than controls.

Symptom patterns and severity scores were available for 24 out of 25 FND patients (Table 1). Of the patients, 17 reported pain symptoms (headache $n=$ 13 , legs $n=3$, body $n=5$ ) with a mean duration of 54.5 (s.D. $=64.2$ ) months. Of the patients, 13 had positive motor symptoms (myoclonic jerks $n=2$, tremor 
Table 1. Patient characteristics

\begin{tabular}{|c|c|c|c|c|c|c|c|}
\hline & \multicolumn{2}{|c|}{$\mathrm{FND}^{\mathrm{a}}$} & \multicolumn{2}{|c|}{$\mathrm{HV}$} & \multirow[b]{2}{*}{ Statistic } & \multirow[b]{2}{*}{$\mathrm{df}$} & \multirow[b]{2}{*}{$p$} \\
\hline & $n$ & Mean (S.D.) & $n$ & Mean (S.D.) & & & \\
\hline Gender, $n$ & & & & & $\chi^{2}=3.20$ & 1 & $>0.07$ \\
\hline Women & 22 & & 17 & & & & \\
\hline Men & 3 & & 7 & & & & \\
\hline Age, years & 25 & $41.6(12.2)$ & 24 & $40.7(15.5)$ & $t=0.23$ & 47 & $>0.07$ \\
\hline BDI-II & 23 & $22.6(10.8)$ & 20 & $6.45(6.1)$ & $t=6.13$ & 35.6 & $<0.001$ \\
\hline STAI & 22 & $45.4(14.2)$ & 20 & $37.5(9.9)$ & $t=2.08$ & 37.6 & 0.041 \\
\hline Pain & 17 & $2.6(0.7)$ & & & & & \\
\hline Positive motor symptoms & 13 & $2.1(0.5)$ & & & & & \\
\hline Negative motor symptoms & 20 & $3.2(0.8)$ & & & & & \\
\hline Non-epileptic seizures & 10 & $3.0(1.0)$ & & & & & \\
\hline Sensory symptoms & 19 & $2.0(0.7)$ & & & & & \\
\hline
\end{tabular}

FND, Functional neurological disorder; HV, healthy volunteers; s.D., standard deviation; df, degrees of freedom; BDI-II, Beck Depression Inventory-II; STAI, Spielberger State-Trait Anxiety Inventory.

${ }^{a}$ FND symptom severity scores were available in 24 patients and were rated from 1 (mild) to 4 (very severe).

$n=7$, dystonia $n=3$, gait abnormality $n=3$ ), and 20 patients reported negative motor symptoms of weakness (lower extremities $n=7$ or upper and lower extremities, $n=13$ ). Average duration of motor symptoms was 53.5 (S.D. $=44.2$ ) months (omission in one subject). Of the patients, 19 stated sensory symptoms (loss of sensation, numbness, pins and needles $n=14$, tinnitus $n=3$, hearing loss $n=1$, double vision $n=3$, blurred vision $n=8$, loss of vision $n=2$ ) with a mean duration of 46.8 (S.D. $=42.6$ ) months (omission in five subjects). Other symptoms such as stutter $(n=4)$, dysarthria $(n=8)$, dysphonia $(n=4)$, swallowing $(n=8)$, memory $(n=10)$, gastrointestinal $(n=6)$, genitourinary $(n=10)$ and cardiovascular symptoms $(n=10)$ were also reported. Most participants had overlapping symptom profiles. For instance, when considering only positive motor, non-epileptic or weakness symptoms, 16 had mixed symptoms, four had only weakness, two had only non-epileptic seizures and two had only positive symptoms. Of the patients, 12 had a symptom affecting the left upper limb they were using to button press. Eight patients had a symptom affecting the right upper limb that they were using to indicate $\mathrm{W}$ or $\mathrm{M}$ with a mouse. All subjects were watched carefully to ensure that the symptoms did not interfere with their capacity to perform the experiment. One might also anticipate that the symptom might similarly affect both $W$ and $M$ which were contrasted.

Two participants had current depression of mild severity, 10 additional subjects had a history of depression, two had panic disorder and two had a history of obsessive-compulsive disorder. Medication use included antidepressants $(n=17)$, pregabalin $(n=5)$, gabapentin $(n=1)$, lamotrigine $(n=2)$, topiramate $(n=1)$ and a synthetic opioid $(n=1)$.

\section{Behavioural results}

Average latencies of the $\mathrm{W}$ and $\mathrm{M}$ judgements relative to the recorded button press were calculated for each individual after excluding outlier trials ( $>2$ s.D. from the individual mean). There was no significant difference in the number of excluded trials in $\mathrm{HV}$ and FND patients (mean 3.7, S.D. $=1.2$ v. 4.1, S.D. $=1.1, p=$ 0.21). Two outlier FND patients ( $>3$ s.D. from mean across both groups) were excluded from the behavioural data analysis, thus 22 FND patients and 24 HVs were included in behavioural data analysis.

A mixed-design analysis of variance (ANOVA) with the factors judgement (W, M) and group (FND, HV) showed significant main effects of group $\left(F_{1,45}=\right.$ 16.54, $p<0.001)$ and judgement $\left(F_{1,45}=33.79, p<\right.$ $0.001)$, and a significant interaction $\left(F_{1,45}=4.38, p=\right.$ 0.042). FND patients showed delayed W [FND -99 (S.D. $=125) \mathrm{ms}$, 95\% confidence interval (CI) -163 to $-35 \mathrm{~ms}$; $\mathrm{HV}-252$ (s.D. $=175$ ) $\mathrm{ms}, 95 \% \mathrm{CI}-315$ to $-190 \mathrm{~ms}$ ] and $\mathrm{M}$ [FND -15 (s.D. $=79$ ) $\mathrm{ms}, 95 \% \mathrm{CI}$ -43 to $+14 \mathrm{~ms}$; HV -72 (S.D. $=56$ ) $\mathrm{ms}, 95 \% \mathrm{CI}-100$ to $-44 \mathrm{~ms}$ ] judgements compared with HV. The interval between $\mathrm{W}$ and $\mathrm{M}$ judgements, $\mathrm{W}-\mathrm{M}$, was used as an implicit measure of conscious awareness of volitional intention. An independent-samples $t$ test demonstrated that FND patients exhibited a significantly reduced $\mathrm{W}-\mathrm{M}$ interval compared with $\mathrm{HV}$ 
$[$ FND -76 (s.D. $=80) \mathrm{ms} ; \mathrm{HV}-180$ (s.D. $=180) \mathrm{ms}, t_{32.3}=$ 2.57, $p=0.015]$.

Individual standard deviations across trials were compared which assess the reporting precision of the $\mathrm{W}$ judgement. FND subjects reported the $\mathrm{W}$ judgement (average S.D. $=156 \mathrm{~ms}$ ) more precisely than HV (average S.D. $\left.=305 \mathrm{~ms}, t_{42.3}=-2.61, p=0.013\right)$, but not the $\mathrm{M}$ judgement (FND average s.D. $=135 \mathrm{~ms} ; \mathrm{HV}$ average S.D. $\left.=80 \mathrm{~ms}, t_{27.9}=1.95, p=0.06\right)$.

Exploratory one-way ANOVAs were used to compare the $\mathrm{W}-\mathrm{M}$ measure of FND patients divided as a function of neurological symptom presentation with $\mathrm{HV}$, showing significant differences for all symptom categories (Table 2). Post-hoc analyses showed that FND patients with any kind of motor symptoms had more reduced $\mathrm{W}-\mathrm{M}$ intervals than $\mathrm{HV}$. To assess the specificity of these findings we compared those with only negative motor symptoms, positive symptoms (with or without negative) and HV. The one-way ANOVA showed a trend $(p=0.052)$; the post-hoc analysis showed that patients with positive motor symptoms differed from $\mathrm{HV}(p=0.019)$, but not those with only negative symptoms $(p>0.2)$ (Bonferroni correction $p<0.025)$. Note that all subjects with positive symptoms also had negative symptoms. We attempted to examine laterality for positive motor symptoms but only three subjects had positive symptoms that did not affect the hand used in the task. We compared subjects with bilateral/left-sided weakness $[n=8,-49$ (s.D. $=52)$ $\mathrm{ms}]$ and right-sided weakness $[n=8,-82($ s.D. $=75) \mathrm{ms}]$ with HV $[n=20,-178$ (s.D. $=174)$ ms], which showed a trend using a one-way ANOVA $(p=0.054)$.

\section{fMRI results}

We excluded two FND patients and two HV from the analysis due to excessive head movement $(>0.5 \mathrm{~mm})$, where over one-third of volumes needed repairing with ArtRepair (Mazaika et al. 2007). Data from 23 FND patients and $22 \mathrm{HV}$ were included in the fMRI analysis; in the comparison between groups, data were also analysed after excluding the two outlier patients identified in the behavioural analysis, and all fMRI findings were consistently observed.

Across all participants, attention to intention relative to attention to movement was associated with increased activity in the bilateral IPL, dlPFC/inferior frontal gyrus and pre-SMA/dIPFC, as well as some minor clusters within temporal and occipital lobes (Fig. 2a, online Supplementary Table S1).

When comparing intention $v$. movement trials between groups, FND patients exhibited significantly reduced activity within the right IPL [supramarginal gyrus, Brodmann area (BA) 40; peak voxel reported in MNI coordinates $x$ y $z$ in $\mathrm{mm}$ : 54, $-32,42$; cluster size =36; $Z=3.58]$ compared with HV (Fig. $2 b$ ). The above-mentioned results were replicated after excluding the two outlier patients: FND patients showed significantly reduced IPL activity with the same peak coordinates (cluster size $=43 ; Z=3.70$ ) compared with HV. An additional activation in the left premotor cortex was also revealed (superior frontal gyrus; MNI coordinates: $-18,6$, 50; cluster size $=49 ; Z=3.67$ ).

The $\mathrm{W}-\mathrm{M}$ interval was positively correlated with BOLD activity in intention $v$. movement trials in the bilateral IPL and primary motor/premotor areas, and left dIPFC and precuneus (Fig. 2c, online Supplementary Table S2).

\section{rsfMRI results}

Data from 25 FND patients and $70 \mathrm{HV}$ [40 women, 40.19 (s.D. = 12.70) years old], including those HV that took part in the fMRI study, were included in the rsfMRI analysis. Compared with HV, FND patients demonstrated reduced connectivity between the right IPC and frontal control regions [dlPFC, anterior cingulate cortex (ACC), BA 10; Fig. 3b], but increased functional connectivity with the premotor cortex and SMA (Fig. 3a, online Supplementary Table S3).

\section{Discussion}

We show using Libet's clock task (Libet et al. 1983) that FND is characterized by impaired awareness of voluntary motor intention with delayed $\mathrm{W}$ relative to $\mathrm{M}$ judgements. The judgement of $\mathrm{W}$ was both abnormally delayed and more precise relative to HV. On an exploratory basis, this delay in $\mathrm{W}$ was particularly evident in patients with involuntary functional positive motor symptoms and less so in those with only negative motor symptoms. Our results concur with a previous report of abnormalities in the conscious experience of action in a small sample of 11 patients with psychogenic tremor (Edwards et al. 2011), replicating these findings in a larger sample. These findings might also explain observations of impaired effortful voluntary movement in FND (Criswell et al. 2010) and common observations of enhanced attention towards both voluntary and involuntary movement. As expected, across all subjects, the attention to intention $v$. movement was associated with enhanced activity in the bilateral IPL, dlPFC and pre-SMA. Crucially, FND subjects had lower activity in the right IPL (BA 40, supramarginal gyrus) compared with HV in the contrast of $\mathrm{W}-\mathrm{M}$. This region was also positively correlated with the behavioural measure $\mathrm{W}-\mathrm{M}$ across all subjects, thus emphasizing its role in the conscious awareness of volitional intention. The network implicated in voluntary motor intention also showed aberrant 
Table 2. W and $M$ judgements as a function of functional symptom type ${ }^{a}$

\begin{tabular}{|c|c|c|c|c|c|c|c|c|}
\hline \multirow[b]{2}{*}{ Symptom } & \multicolumn{2}{|c|}{ Present } & \multicolumn{2}{|c|}{ Absent } & \multicolumn{2}{|l|}{$\mathrm{HV}$} & \multirow[b]{2}{*}{ Present $v$. HV: $p$} & \multirow{2}{*}{$\begin{array}{l}\text { Absent } v . \\
\mathrm{HV}: p\end{array}$} \\
\hline & $n$ & Mean (s.D.) & $n$ & Mean (S.D.) & Mean (S.D.) & $F(p)$ & & \\
\hline Positive motor & 12 & $-55(78)$ & 10 & $-87(66)$ & $-180(180)$ & $3.58(0.037)$ & 0.017 & 0.100 \\
\hline Negative motor & 18 & $-79(78)$ & 4 & $-26(25)$ & $-180(180)$ & $3.69(0.033)$ & 0.029 & 0.051 \\
\hline $\begin{array}{l}\text { Positive motor } v \text {. } \\
\text { only negative } \\
\text { motor }\end{array}$ & 12 & $\begin{array}{l}\text { Positive (with/ } \\
\text { without negative) } \\
-55(78)\end{array}$ & 8 & $\begin{array}{l}\text { Negative } \\
\text { only } \\
-101(67)\end{array}$ & $-180(180)$ & $3.18(0.052)$ & $\begin{array}{l}\text { Positive (with/ } \\
\text { without negative) } \\
0.019\end{array}$ & $\begin{array}{l}\text { Negative } \\
\text { only } \\
0.211\end{array}$ \\
\hline $\begin{array}{l}\text { Non-epileptic } \\
\text { seizures }\end{array}$ & 8 & $-49(64)$ & 14 & $-79(78)$ & $-180(180)$ & $3.55(0.037)$ & 0.037 & 0.040 \\
\hline Somatosensory & 13 & $-81(63)$ & 9 & $-53(85)$ & $-180(180)$ & $3.55(0.038)$ & 0.054 & 0.027 \\
\hline
\end{tabular}

Data are given as mean difference between $\mathrm{W}$ and $\mathrm{M}$ judgements (in ms) (S.D.).

HV, Healthy volunteers; S.D., standard deviation; ANOVA, analysis of variance; df, degrees of freedom.

${ }^{a} F$ values of the ANOVAs are given, as well as $p$ values of the ANOVAs and Fisher's least significant difference post-hoc tests. All ANOVAs had $\mathrm{df}_{\text {factor }}=2$ and $\mathrm{df}_{\text {error }}=43$, except the ANOVA comparing functional neurological disorder patients with positive motor symptoms, patients with only negative symptoms and $\mathrm{HV}$, which had $\mathrm{df}_{\text {factor }}=2$ and $\mathrm{df}_{\text {error }}=41$.

(a)

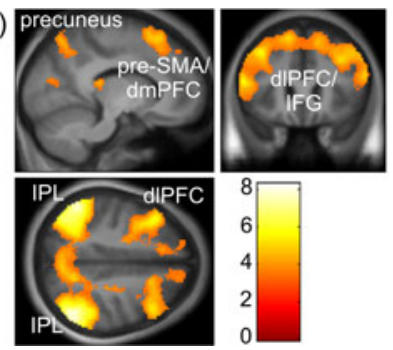

(b)

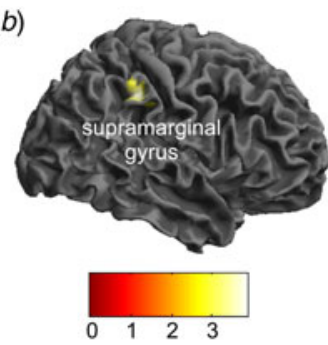

(c)

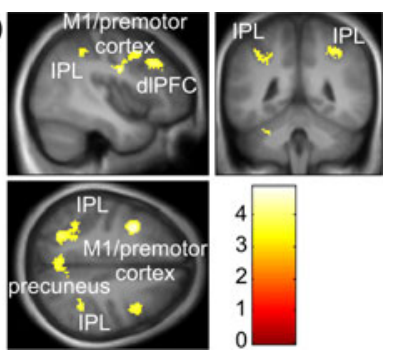

Fig. 2. Attention to intention $v$. movement. (a) Significant activations associated with attention to intention compared with attention to movement (intention $v$. movement contrast) across all participants $(n=45)$. (b) Regions of significantly decreased activity for functional neurological disorder patients $(n=23)$ compared with healthy volunteers $(n=22)$ when attending to intention compared with attending to movement. Image displayed at $p<0.005$ (uncorrected) for illustration. (c) Results of the correlation between the intention $v$. movement contrast and the behavioural measure W-M across all participants $(n=45)$. Image displayed at $p<0.001$ (uncorrected) for illustration. pre-SMA, Pre-supplementary motor area; dmPFC, dorsomedial prefrontal cortex; IPL, inferior parietal lobule; dIPFC, dorsolateral prefrontal cortex; IFG, inferior frontal gyrus; M1, primary motor cortex.

functional connectivity at rest in FND subjects. FND showed reduced resting-state functional connectivity between the right IPC and prefrontal structures (dIPFC, ACC, BA 10), regions upstream in the processing of intention and action selection, but increased connectivity with the premotor cortex and SMA, regions downstream in the processing of intention and motor preparation. Previous studies in FND have implicated lower TPJ/IPC activity to involuntary functional symptoms (Voon et al. 2010) and lower SMA activity during voluntary motor preparation (Voon et al. 2011). Here we specifically isolate the awareness of voluntary intention and build on data implicating an abnormal fronto-parietal network and suggest that these findings might play a role in the subjective sense of involuntariness.
Our findings may implicate a general impairment in inferior parietal function. Previous studies have shown hypoactivity of the right TPJ in the comparison of involuntary and mimicked tremor (Voon et al. 2010). As neural regions associated with the sensory outcome appeared to be intact, we had speculated that the primary deficit might be related to an impairment at the level of the sensory prediction (Voon et al. 2010). A follow-up study further showed decreased functional connectivity of the right TPJ seed using average coordinates from a meta-analysis of self-agency studies (peak $x$ $y z=51,-46,31 \mathrm{~mm}$ ) within BA 39 (Maurer et al. 2016). The TPJ is a large region encompassing the IPL. The right IPL activation in the attention to intention $v$. movement condition across all subjects was very large (cluster size $k=10439$ ) and included the right TPJ. However, the 

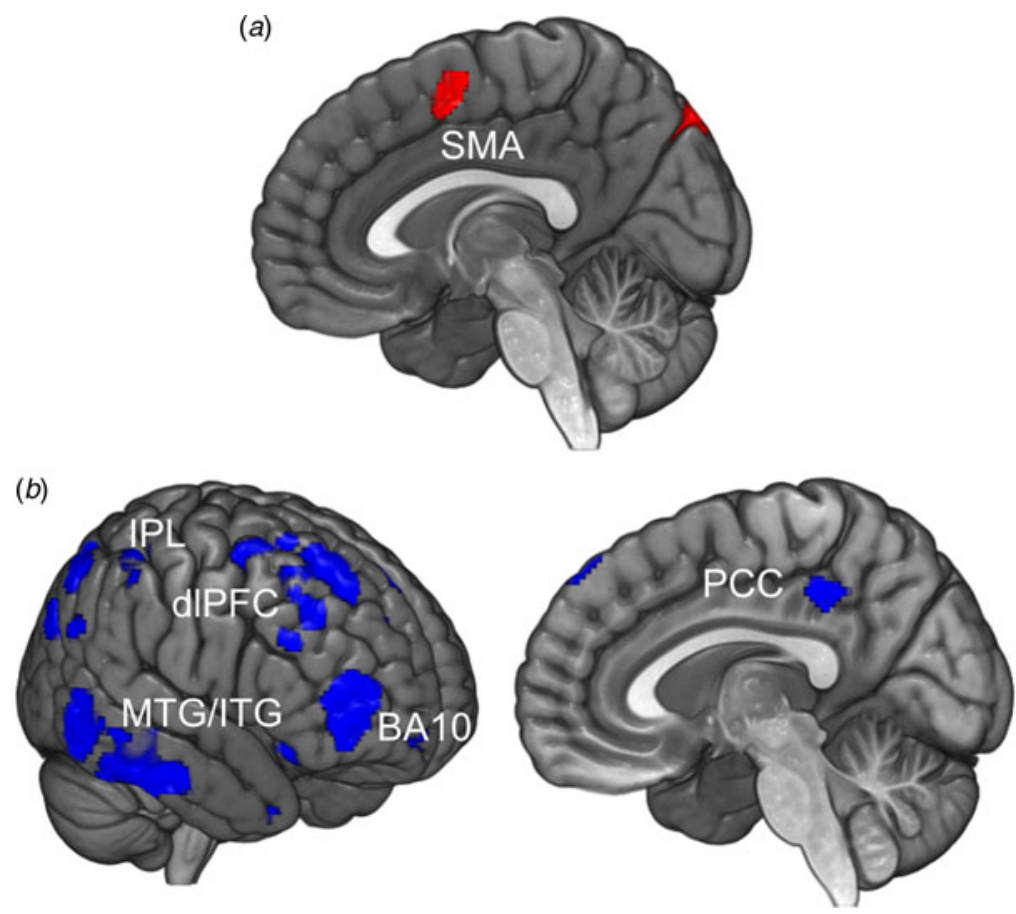

Fig. 3. Resting-state functional connectivity from right inferior parietal cortex (IPC) seed. (a) Increased (functional neurological disorder patients $>$ healthy volunteers; FND $>\mathrm{HV}$ ) and $(b)$ decreased (HV $>$ FND) functional connectivity from IPC to whole brain for FND patients $(n=25)$ compared with HV $(n=70)$ during rest. Image displayed at $p<0.005$ (uncorrected) for illustration. SMA, Supplementary motor area; IPL, inferior parietal lobule; dlPFC, dorsolateral prefrontal cortex; MTG, middle temporal gyrus; ITG, inferior temporal gyrus; BA, Brodmann area; PCC, posterior cingulate cortex.

right IPL activation (peak $x y z=54,-32,42 \mathrm{~mm}$ ) in the group comparison (FND $<\mathrm{HV}$; Figs 2 and 3 ) is localized in the angular/supramarginal gyrus in BA 40 and distant from the superior temporal lobule. Both BA 39 and BA 40 are implicated in impairments in intention using Libet's clock (Desmurget \& Sirigu, 2012). A generalized impairment in inferior parietal cortical function may be an issue which has been observed in some but not all FND studies (for a review, see Voon et al. 2016). Differences in imaging task design and hence function might thus highlight different parietal regions. Thus, although both studies implicate impairments in the IPC, the focus on intention in voluntary movements implicates BA 40 whereas differences between the comparison of involuntary and voluntary movement might highlight more comparator systems of sensory prediction and outcome implicating BA 39 or the TPJ.

\section{Mechanistic considerations}

There are several possible explanations for our findings. Here we discuss plausible mechanisms including the role of attention, impairments in explicit intentional processes, decision thresholds and movement veto.

Attending to and reporting the intention to move might be more difficult and demanding more attention than reporting the movement itself, so that subjects with attentional impairments may have more difficulty in making the $\mathrm{W}$ judgement. Non-specific impairments in attention have been documented across some but not all studies in FND (for a review, see Voon et al. 2016). However, in our study, FND patients reported the $\mathrm{W}$ judgement more precisely than $\mathrm{HV}$, thus suggesting that the reduced latency is unlikely to be due to a non-specific attentional impairment. We also controlled for non-specific attention effects by comparing two conditions that require the allocation of attention to either intention or movement. An intriguing follow-up of this study might be to consider how $\mathrm{W}-\mathrm{M}$ might present in subjects with malingering in which the symptoms are deliberately produced. One might speculate that the variability associated with reporting of $\mathrm{W}$ might be much greater than observed in FND.

Theoretical models and empirical evidence suggest enhanced directed attention towards self-related representations and pathological symptoms (Brown, 2004; Cojan et al. 2009; Edwards et al. 2012). These findings of an enhanced precision (inverse variance) of abnormally delayed voluntary intention converge with theories of enhanced attention towards and thus precision of abnormal pathological priors or expectations in 
FND (for a review, see Edwards et al. 2012). Although enhanced attention to the movement symptom is observed in FND, what is not clear is whether this is a primary pathology or possibly a compensatory mechanism underlying primary impairments in volitional processes. Enhanced attention to both voluntary and functional movements is observed clinically in FND (van Poppelen et al. 2011) and attention appears to play a role in the expression of the symptom as demonstrated by clinical signs of improvement or cessation of functional symptoms with distractability or shifts of attention away from the functional movement (Schwingenschuh \& Deuschl, 2016). Attention focused on a novel voluntary movement is required for optimal motor function but can impair automatic overlearned habitual movements. That attention is enhanced to self in FND might be compensatory and secondarily related to primary impairments in voluntary motor intention. The enhanced attention might then also interfere with skilled automatic movements. That focused attention increases the functional movement suggests that the aberrant movement is novel and goal-oriented in nature rather than automatic and habitual as otherwise, attention should impair habits and decrease the functional movement. Our findings would be consistent with clinical observations of increased attention towards both the involuntary and voluntary movements in FND.

Several lines of evidence suggest impairments in explicit but not implicit motor processes in FND, suggesting possible impairments in motor conceptualization or volition, first theorized by Spence et al. (2000) (for a review, see Voon et al. 2016). FND subjects are impaired at explicit instructed $v$. implicit automatic mental rotation of hands or feet (Roelofs et al. 2001). In this study, functional paralysis subjects subjectively reported that they could not explicitly mentally rotate their hands or feet to match the image in $51 \%$ of foot trials, compared with $0 \%$ in controls. Similarly, FND subjects were impaired in tasks dissociating explicit pre-planned movements under full control or under greater certainty but intact in implicit movements occurring more automatically under contexts in which subjects may be less aware (e.g. one-back reaching, visuomotor transformation or variable predictive value of precued reaction time) (Parees et al. 2013). Focusing on the motor intention phase of movement, FND subjects also show decreased activity in the SMA and increased activity in limbic regions (amygdala, anterior insula) relative to healthy controls (Voon et al. 2011). FND was also associated with decreased functional connectivity between the dIPFC and SMA in freely chosen $v$. directed cued movement, suggesting a possible impairment in higher-order action selection during freely chosen actions.
Together these findings suggest primary impairments in explicit motor intentional processes with intact implicit automatic processes. These findings help explain observations of impaired voluntary movement in FND as tested on finger tapping (Criswell et al. 2010) and common clinical observations of enhanced attention and effort directed towards voluntary movement which may be secondary to the primary impairment. Indeed, a supportive clinical criterion of functional movement is slowed effortful voluntary movement (Schwingenschuh \& Deuschl, 2016). The mechanisms underlying functional movement may also represent an extension of this impairment in explicit volitional motor process engaging enhanced attention. The enhanced attention may also then secondarily interfere with skilled automatic movements.

These abnormalities in intention awareness appear to be greater in patients with positive motor symptoms, although we note that the relationship with specific neurological symptoms should be interpreted cautiously given the limited sample size. Nevertheless, several intriguing mechanisms may be specifically relevant to positive motor symptoms. It has been suggested that movements are produced when a certain decision threshold is crossed and that the precise moment at which this threshold is crossed is largely determined by spontaneous subthreshold fluctuations of neuronal activity (Schurger et al. 2012). In the context of FND, it could be speculated that patients might have a lower decision threshold and/ or increased neuronal subthreshold fluctuations, resulting in an increased likelihood of abnormally crossing said threshold and thus producing aberrant movements. Indeed, the Bereitschaftspotential, which has been posited to reflect the increase in spontaneous neuronal subthreshold fluctuations preceding voluntary movements (Schurger et al. 2012), has been found to precede psychogenic myoclonus (Terada et al. 1995).

Another possible underlying mechanism could be related to the interval between awareness of intention and movement, which has been hypothesized to temporally allow for a conscious inhibition or veto of the movement (Libet, 1999; Haggard \& Libet, 2001), as well as for an evaluation of whether the selected action might be optimal to obtain the desired effect (Haggard \& Libet, 2001). A recent study (Schultze-Kraft et al. 2016) reported that initiated movements could be inhibited if a stop signal occurred up to $200 \mathrm{~ms}$ prior to movement onset, but not if it occurred under that time. Since the temporal shift of intention awareness towards the movement in FND patients is well under $200 \mathrm{~ms}$, it could suggest that this results in a decrease in the time available and likelihood of action inhibition or movement veto. Still, this remains speculative based 
solely on the data at hand. However, as in the current study, shortened W-M intervals have been mainly observed in neurological and neuropsychiatric disorders characterized by positive symptomatology (Edwards et al. 2011; Moretto et al. 2011; Tabu et al. 2015). These findings converge with a study of FND focusing on positive motor symptoms in which the only cognitive deficit observed across a range of tasks was impaired response inhibition on a Go/ NoGo task (Voon et al. 2013). Alternatively, the findings may also be related to prior experience with functional movements, which may secondarily result in impaired reliance on the subjective awareness of the intention to move.

\section{IPC}

Our findings implicate the IPC, but, contrary to our hypothesis, not the SMC. Cortical stimulation of the IPC (BA 39/40) has been associated with subjective feelings of 'wanting to move' a body part, whereas stimulation of the SMC results in an uncontrollable 'urge' to produce a specific movement (Desmurget et al. 2009; Desmurget \& Sirigu, 2012). This phenomenological difference suggests the intentional feelings evoked in the IPC may lie upstream of the SMC, and has been hypothesized to reflect the role of the IPC in pure intention (Desmurget et al. 2009). However, which of these structures is key to voluntary action remains controversial. Some authors attribute this role to the pre-SMA, highlighting its involvement in transforming thoughts into actions (Haggard, 2008), whereas others emphasize evidence suggesting that the locus of consciousness might be within posterior brain regions (Koch et al. 2016). Damage to both frontomesial and IPC regions has been associated with the alien hand syndrome, in which movements are expressed outside of volitional control (Scepkowski \& Cronin-Golomb, 2003). In an fMRI study of a patient with a posterior parietal lesion, alien hand movement was associated with circumscribed activity of the contralateral primary motor cortex, which was suggested to arise in the absence of volitional awareness from the IPC (Assal et al. 2007). Indeed, in patients with IPC stroke lesions, the latency of the W judgement is shifted towards movement onset as compared with HV and cerebellar patients (Sirigu et al. 2004). Together these findings suggest a role for the IPC at an early stage of motor intention.

Another possible parallel lies in studies of motor imagery or internal rehearsal of motor representations. Recently, a tetraplegic patient was shown to be able to control posterior parietal activity though motor imagery (Aflalo et al. 2015). Recordings showed that neurons in this area code both goal and imagined trajectory of the movements, indicating that this region encodes motor intention and that its signals could be used for neuroprosthetic applications (Aflalo et al. 2015). Motor imagery implicates similar neural regions as action execution (Decety et al. 1994; Sirigu et al. 1996) and has been studied in FND as discussed above. In a limb rotation task (Roelofs et al. 2001), participants with functional paralysis showed no difference to HV when judging whether images of rotated hands and feet corresponded to the right or left limb. However, they were slower than HV when explicitly asked to mentally rotate their own limbs to match the position of the image, with patients subjectively reporting impaired motor imagery in that they had difficulty mentally rotating their limbs to match the image whereas no difficulties were reported in controls. These impairments in explicit motor imagery prior to and without actual movement led the authors to emphasize an impairment in intentional processes in FND (Roelofs et al. 2001).

\section{Limitations and conclusion}

The study is not without limitations. Although we specifically recruited subjects that were able to remain still in the MRI environment, it cannot be completely ruled out that the subjects' neurological symptoms interfered with testing and that weakness or functional movements occurred during scanning. However, as these symptoms may have occurred randomly across the blocks, the contrast used should effectively cancel any neural activity from adventitious symptoms. Larger sample sizes with greater symptom specificity would also be helpful in understanding the relationship of this measure with specific symptoms. Furthermore, how these findings might be influenced by other symptoms commonly observed in FND including panic symptoms or other somatoform disorders remains to be established. The majority of our sample was also on medications, which is commonly observed in FND cohorts. The use of medications might interfere with imaging findings; further studies in other samples on such chronic medications might be indicated. Co-morbid symptoms of depression or anxiety are also very common in FND, and the effect of depression or anxiety symptoms cannot be ruled out. When BDI and STAI are added as covariates in ANOVA in behaviour analysis, significant interactions between conditions (intention or movement) $v$. BDI or STAI were observed and the main effect of groups or conditions was no longer observed. FND is also commonly associated with generalized anxiety which was not assessed in this study.

Despite the main findings of Libet et al. (1983) having been widely replicated (Lau et al. 2004; Edwards 
et al. 2011; Fried et al. 2011), including in the current study, Libet's clock task has not been exempt of criticism. The precision and nature of the $\mathrm{W}$ judgement has been called into question, with authors suggesting that the high attentive demands of the task might result in an imprecise measurement of the internal process (Stamm, 1985), or that the $\mathrm{W}$ judgement might represent a peak of intention (Ringo, 1985), meaning that the subject's intention would in reality precede the reported urge, in contrast to the simultaneity posited by Libet et al. (1983). Recent studies suggest that the decision for a movement does not abruptly appear in a binary manner but is gradually constructed; thus Libet's task may involve a cut-off for binary translation of the continuous preparation of action decision (Guggisberg \& Mottaz, 2013). In addition, that subjective time of intention onset is influenced by perceptual information such as sensory feedback (Guggisberg \& Mottaz, 2013; Wolpe \& Rowe, 2014). Introspection to one's own intention might be also intermittent or rather retrospectively inferred. Critics thus implied that the unconscious nature of the movement intention, as reflected by the Bereitschaftspotential preceding intention awareness, would not be such (Stamm, 1985). Haggard \& Libet (2001), however, countered that even the largest estimates of the prior entry effect, by which attention affects the judgement of synchrony of events occurring in different streams, are an order of magnitude smaller than the $200 \mathrm{~ms}$ interval between Bereitschaftspotential and $\mathrm{W}$ judgement posited in the original work (Libet et al. 1983). Moreover, even those who questioned the precision or validity of the absolute $\mathrm{W}$ and/or $\mathrm{M}$ judgements conceded that their criticism might not affect the relative distance between them (Rollman, 1985). The latter is of particular importance to the current work, as it is this interval that is postulated to be used by the subject to monitor the desirability and effect of the action (Libet's veto) and whether it is an optimal action plan (Haggard's specificity) (Haggard \& Libet, 2001). Therefore, irrespective of the conscious nature of the movement, a reduced veto period would have a higher likelihood of resulting in maladaptive actions, such as the aberrant behaviours observed in our FND patients and reported by previous researchers (Edwards et al. 2011; Moretto et al. 2011; Tabu et al. 2015).

In conclusion, our findings build on reports suggesting impairments in motor intention awareness in FND and specifically highlight abnormalities of the early motor processing network focusing on the right IPL. These findings might explain common clinical observations of enhanced attention towards movements in FND and impaired effortful voluntary movements and provide possible novel targets for therapeutic intervention that might include psychological or physiological interventions or neuromodulation.

\section{Supplementary material}

The supplementary material for this article can be found at https://doi.org/10.1017/S0033291717000071

\section{Acknowledgements}

N.D. is a research fellow of the Deutsche Forschungsgemeinschaft (DO1915/1-1). V.V. is a Wellcome Trust Intermediate Fellow (983705/Z/10/Z). The study was funded by the Biomedical Research Council. Funders had no role in the study design, in the collection, analysis and interpretation of the data, in the writing of the report, or in the decision to submit the paper for publication.

\section{Declaration of Interest}

None.

\section{References}

Aflalo T, Kellis S, Klaes C, Lee B, Shi Y, Pejsa K, Shanfield K, Hayes-Jackson S, Aisen M, Heck C, Liu C, Andersen RA (2015). Decoding motor imagery from the posterior parietal cortex of a tetraplegic human. Science 348, 906-910.

American Psychiatric Association (2013). Diagnostic and Statistical Manual of Mental Disorders. American Psychiatric Association: Washington, DC.

Anderson KE, Gruber-Baldini AL, Vaughan CG, Reich SG, Fishman PS, Weiner WJ, Shulman LM (2007). Impact of psychogenic movement disorders versus Parkinson's on disability, quality of life, and psychopathology. Movement Disorders 22, 2204-2209.

Assal F, Schwartz S, Vuilleumier P (2007). Moving with or without will: functional neural correlates of alien hand syndrome. Annals of Neurology 62, 301-306.

Behzadi Y, Restom K, Liau J, Liu TT (2007). A component based noise correction method (CompCor) for BOLD and perfusion based fMRI. NeuroImage 37, 90-101.

Beck AT, Ward CH, Mendelson MM, Mock JJ, Erbaugh JJ (1961). An inventory for measuring depression. Archives of General Psychiatry 4, 561-571.

Brass M, Haggard P (2007). To do or not to do: the neural signature of self-control. Journal of Neuroscience 27, 91419145.

Brown RJ (2004). Psychological mechanisms of medically unexplained symptoms: an integrative conceptual model. Psychological Bulletin 130, 793-812.

Carson AJ, Ringbauer B, Stone J, McKenzie L, Warlow C, Sharpe M (2000). Do medically unexplained symptoms matter? A prospective cohort study of 300 new referrals to neurology outpatient clinics. Journal of Neurology, Neurosurgery and Psychiatry 68, 207-210. 
Cojan Y, Waber L, Carruzzo A, Vuilleumier P (2009). Motor inhibition in hysterical conversion paralysis. NeuroImage 47, 1026-1037.

Criswell S, Sterling C, Swisher L, Evanoff B, Racette BA (2010). Sensitivity and specificity of the finger tapping task for the detection of psychogenic movement disorders. Parkinsonism Related Disorders 16, 197-201.

Decety J, Perani D, Jeannerod M, Bettinardi V, Tadary B, Woods R, Mazziotta JC, Fazio F (1994). Mapping motor representations with positron emission tomography. Nature 371, 600-602.

Desmurget M, Reilly KT, Richard N, Szathmari A, Mottolese C, Sirigu A (2009). Movement intention after parietal cortex stimulation in humans. Science 324, 811-813.

Desmurget M, Sirigu A (2012). Conscious motor intention emerges in the inferior parietal lobule. Current Opinion in Neurobiology 22, 1004-1011.

Edwards MJ, Adams RA, Brown H, Pareés I, Friston KJ (2012). A Bayesian account of 'hysteria'. Brain 135, 34953512.

Edwards MJ, Moretto G, Schwingenschuh P, Katschnig P, Bhatia KP, Haggard P (2011). Abnormal sense of intention preceding voluntary movement in patients with psychogenic tremor. Neuropsychologia 49, 2791-2793.

Fried I, Katz A, McCarthy G, Sass KJ, Williamson P, Spencer SS, Spencer DD (1991). Functional organization of human supplementary motor cortex studied by electrical stimulation. Journal of Neuroscience 11, 3656-3666.

Fried I, Mukamel R, Kreiman G (2011). Internally generated preactivation of single neurons in human medial frontal cortex predicts volition. Neuron 69, 548-562.

Guggisberg AG, Mottaz A (2013). Timing and awareness of movement decisions: does consciousness really come too late? Frontiers in Human Neuroscience 7, 385.

Haggard P (2008). Human volition: towards a neuroscience of will. Nature Reviews. Neuroscience 9, 934-946.

Haggard P, Libet B (2001). Conscious intention and brain activity. Journal of Consciousness Studies 8, 47-64.

Hallett M (2010). Physiology of psychogenic movement disorders. Journal of Clinical Neuroscience 17, 959-965.

Koch C, Massimini M, Boly M, Tononi G (2016). Neural correlates of consciousness: progress and problems. Nature Reviews. Neuroscience 17, 307-321.

Kundu P, Inati SJ, Evans JW, Luh W-M, Bandettini PA (2012). Differentiating BOLD and non-BOLD signals in fMRI time series using multi-echo EPI. NeuroImage 60, 1759-1770.

Lau HC, Rogers RD, Haggard P, Passingham RE (2004). Attention to intention. Science 303, 1208-1210.

Libet B (1999). Do we have free will? Journal of Consciousness Studies 6, 47-57.

Libet B, Gleason CA, Wright EW, Pearl DK (1983). Time of conscious intention to act in relation to onset of cerebral activity (readiness-potential). Brain 106, 623-642.

Maurer CW, LaFaver K, Ameli R, Epstein SA, Hallett M, Horovitz SG (2016). Impaired self-agency in functional movement disorders: a resting-state fMRI study. Neurology $87,564-570$.
Mazaika PK, Whitfield-Gabrieli S, Reiss AL, Glover GH (2007). Artifact repair for fMRI data from high motion clinical subjects. In Annual Meeting of the Organization of Human Brain Mapping: Chicago, USA.

Moretto G, Schwingenschuh P, Katschnig P, Bhatia KP, Haggard P (2011). Delayed experience of volition in Gilles de la Tourette syndrome. Journal of Neurology, Neurosurgery and Psychiatry 82, 1324-1327.

Parees I, Kassavetis P, Saifee TA, Sadnicka A, Davare M, Bhatia KP, Rothwell JC, Bestmann S, Edwards MJ (2013). Failure of explicit movement control in patients with functional motor symptoms. Movement Disorders 28, 517-523.

Ringo JL (1985). Timing volition: questions of what and when about W. Behavioral and Brain Sciences 8, 550-551.

Roelofs K, Näring GWB, Keijsers GPJ, Hoogduin CAL, Van Galen GP, Maris E (2001). Motor imagery in conversion paralysis. Cognitive Neuropsychiatry 6, 21-40.

Rollman GB (1985). Sensory events with variable central latencies provide inaccurate clocks. Behavioral and Brain Sciences 8, 551-552.

Scepkowski LA, Cronin-Golomb A (2003). The alien hand: cases, categorizations, and anatomical correlates. Behavioral and Cognitive Neuroscience Reviews 2, 261-277.

Schultze-Kraft M, Birman D, Rusconi M, Allefeld C, Görgen K, Dähne S, Blankertz B, Haynes J-D (2016). The point of no return in vetoing self-initiated movements. Proceedings of the National Academy of Sciences of the United States of America 113, 1080-1085.

Schurger A, Sitt JD, Dehaene S (2012). An accumulator model for spontaneous neural activity prior to self-initiated movement. Proceedings of the National Academy of Sciences of the United States of America 109, E2904-E2913.

Schwingenschuh P, Deuschl G (2016). Functional tremor. In Functional Neurological Disorders (ed. M Hallett, J Stone and A Carson), p. 19. Elsevier: Amsterdam.

Sirigu A, Daprati E, Ciancia S, Giraux P, Nighoghossian N, Posada A, Haggard P (2004). Altered awareness of voluntary action after damage to the parietal cortex. Nature Neuroscience 7, 80-84.

Sirigu A, Duhamel J-R, Cohen L, Pillon B, Dubois B, Agid Y (1996). The mental representation of hand movements after parietal cortex damage. Science 273, 1564-1568.

Slotnick SD, Moo LR, Segal JB, Hart Jr. J (2003). Distinct prefrontal cortex activity associated with item memory and source memory for visual shapes. Cognitive Brain Research $17,75-82$.

Spence SA, Crimlisk HL, Cope H, Ron MA, Grasby PM (2000). Discrete neurophysiological correlates in prefrontal cortex during hysterical and feigned disorder of movement. Lancet 355, 1243-1244.

Spielberger CD, Gorsuch RL, Lushene R, Vagg PR, Jacobs GA (1983). Manual for the State-Trait Anxiety Inventory. Consulting Psychologists Press: Palo Alto, CA.

Stamm JS (1985). The uncertainty principle in psychology. Behavioral and Brain Sciences 8, 553-554.

Tabu H, Aso T, Matsuhashi M, Ueki Y, Takahashi R, Fukuyama H, Shibasaki H, Mima T (2015). Parkinson's 
disease patients showed delayed awareness of motor intention. Neuroscience Research 95, 74-77.

Terada K, Ikeda A, Van Ness PC, Nagamine T, Kaji R, Kimura J, Shibasaki H (1995). Presence of Bereitschaftspotential preceding psychogenic myoclonus: clinical application of jerk-locked back averaging. Journal of Neurology, Neurosurgery and Psychiatry 58, 745-747.

van Poppelen D, Saifee TA, Schwingenschuh P, Katschnig P, Bhatia KP, Tijssen MA, Edwards MJ (2011). Attention to self in psychogenic tremor. Movement Disorders 26, 25752576.

Voon V, Brezing C, Gallea C, Hallett M (2011). Aberrant supplementary motor complex and limbic activity during motor preparation in motor conversion disorder. Movement Disorders 26, 2396-2403.

Voon V, Cavanna AE, Coburn K, Sampson S, Reeve A, LaFrance Jr. WC; on behalf of the American
Neuropsychiatric Association Committee for Research (2016). Functional neuroanatomy and neurophysiology of functional neurological disorders (conversion disorder). Journal of Neuropsychiatry and Clinical Neurosciences 28, 168-190.

Voon V, Ekanayake V, Wiggs E, Kranick S, Ameli R, Harrison NA, Hallett M (2013). Response inhibition in motor conversion disorder. Movement Disorders 28, 612-618.

Voon V, Gallea C, Hattori N, Bruno M, Ekanayake V, Hallett M (2010). The involuntary nature of conversion disorder. Neurology 74, 223-228.

Whitfield-Gabrieli S, Nieto-Castañón A (2012). Conn: a functional connectivity toolbox for correlated and anticorrelated brain networks. Brain Connectivity 2, 125-141.

Wolpe N, Rowe JB (2014). Beyond the "urge to move": objective measures for the study of agency in the post-Libet era. Frontiers in Human Neuroscience 8, 450. 